\title{
Qualitative methods to evaluate Mediterranean diet in adults
}

\author{
Mariette Gerber* \\ Groupe d'Epidémiologie Métabolique, Centre de recherche en Cancérologie Centre Régional de Lutte contre le \\ Cancer, Val d'Aurelle-Paul Lamargue, 34298 Montpellier Cedex 5, France
}

\begin{abstract}
Objective: To fulfil a comprehensive approach to consumption, which is necessary to characterise food habits and their relationship to diseases, using a diet quality index (DQI) developed for a Mediterranean region (Med-DQI).

Setting: A cross-sectional nutritional survey provided the data for the construction of the DQI.

Subjects: A representative sample made up of 964 subjects from a French Mediterranean area, Hérault département.

Methods: Foods such as olive oil, fish and cereals were used instead of nutrients to build up scores which constructed the Med-DQI. Biochemical analysis identified biomarkers used to validate the Med-DQI. Correspondence factorial analysis illustrated the characteristics of subjects with different scores given by the Med-DQI. Results: The subjects could be satisfactorily classified by the Med-DQI. The oldest age, less educated, overweight, manual workers, living in a rural area and male classes showed a better Med-DQI. For women, they were also from the oldest age class, but some of them were from the upper social class, with an ideal BMI and lived by the Mediterranean shore. A composite biomarker index, based on plasma carotene and vitamin E levels and the percentage of EPA and DHA in erythrocytes membranes, can identify subjects with good and poor Med-DQI.

Conclusion: Med-DQI G is a good instrument to identify groups at risk with regard to nutritional quality. Subjects with $\alpha+\beta$-carotene levels $>1 \mathrm{mg} / \mathrm{l}$, vitamin $\mathrm{E}>30 \mathrm{mg} / \mathrm{l}$, EPA $>0.65 \%$ and DHA $>4 \%$ of fatty acids in erythrocytes are likely to have a good diet.
\end{abstract}

Keywords Diet Quality Index Diet Profiles Mediterranean diet
It was shown in several countries that the Mediterranean diet is fading away. In the Languedoc-Roussillon (LR) region of Southern France, it was shown ${ }^{1}$ that vegetable and fruit consumption remained similar to the traditional Mediterranean diet ${ }^{2}$, dairy product and meat intakes were too large and olive oil intake too small, resulting in a ratio of monounsaturated fatty acids (MUFA)/saturated fatty acids (SFA) that is too high. The regional population is losing its advantage in terms of cardiovascular diseases (CVD) and cancer incidences compared to the national rates. Moreover, women display a higher CVD rate in LR than in France as a whole. Mediterranean Diet and Health (MEDHEA), an ecological study analysed by factorial analysis, showed that Mediterranean foodstuff consumption was opposed to the incidence of cancers at all sites, and especially stomach and colon-rectum cancers in $\mathrm{LR}^{3}$. This was another reason to investigate whether the Mediterranean food habits were still shared by the regional population. A comprehensive approach of consumption to necessary whether one wishes to characterise food habits or their relationship to diseases ${ }^{4}$. To fulfil this objective, diet quality index (DQI) is a helpful method. We present here the index we developed for a Mediterranean region, together with the although identification of biological markers that validated the DQI, and which could also be used to identify the Mediterranean characteristics of food intake in subjects.

\section{Subjects and methods}

\section{Subjects}

Subjects aged 30-77 years were randomly recruited between January 1994 and November 1996 proportionally to the geographical demography of the region and according to the specificity of subregions: rural, urban, and Mediterranean shore. After exclusion of overreporting and incomplete questionnaires, the final sample consisted of 964 subjects, 473 men and 491 women. Information on food consumption was elicited with a validated frequency questionnaire 5

\section{Construction of the DQI}

The DQI devised by Patterson et al. ${ }^{6}$ was first applied to the Mediterranean sample, but it was inadequate to 
classify the population in that there was no gradient of consumption with increasing scores. This was true for the variables 'total fat' and 'maintain protein intake at moderate levels'. Thus a specific Mediterranean-DQI (Med-DQI) had to be devised. Olive oil was added with a score increasing with a lower intake. Protein was replaced by meat, because fish was added with an opposite gradient. Each nutrient or food group was assigned three scores ( 0,1 and 2 ) on the basis of recommended guidelines where these exist (cholesterol, SFA), or by dividing the population's consumption into tertiles where there was no specific recommendation for the food. The scores are described in Table 1. Scores were totalled for each subject, giving a total score for the DQI. The lower the DQI, the healthier the diet. The best DQI has a score of 0 , the poorest, 14 . We pooled the scores as follows: good, 1-4; medium-good, 5-7; medium-poor, 8-10; poor, 11-14. A complementary score was developed incorporating tobacco consumption (DQI becoming DQI-f) to investigate the interaction of tobacco consumption with food intake and with biomarkers, such as $\beta$ carotene.

\section{Biochemical analysis}

In order to both validate the Med-DQI and identify markers of a good diet, biochemical analyses were performed in plasma and erythrocytes of a subsample of the participants (146, both genders). Plasma cholesterol, triglycerides, vitamins $\mathrm{A}$ and $\mathrm{E}$, and carotenoids were measured. Fatty acids were measured in erythrocytes.

\section{Statistical analysis}

All analyses were performed with SAS (version 6.11; SAS institute, Cary, NC, USA). Significance of difference of median values was estimated using the Wilcoxon test rank. To characterise the subjects in each DQI class, we used a correspondence factorial analysis with the correspondence analysis procedure in SAS. The subjects of each DQI class identified by their scores were analysed against possible food habit determinants: age, body mass index (BMI), tobacco use, wine consumption, alcohol consumption, vitamin and mineral supplement intake, socioprofessional class, education, residence in subregions, rural or urban area. Spearman correlations were computed between the DQI scores and the values of biological variables, and a composite index of these variables. The association of the indexes of two or more biological variables constructed this composite index. For example, the value 1 of the composite index of cholesterol, vitamin $\mathrm{E}$ and carotene consisted in the highest tertile of cholesterol and the lowest tertiles of the two other variables.

\section{Results}

The mean consumption in each of the classes is given in Table 2 . There exists a regular progression in each variable of the score, showing that the construction of the DQI is satisfactory. For cereals, however, the progression is irregular indicating that the assessment of their consumption was not discriminating. Less than $10 \%$ of the population could claim to have preserved the Mediterranean diet, and more than $50 \%$ are characterised by a medium-poor to poor diet.

In order to identify the population classes with the poorest food habits and eventually to adapt specific nutritional recommendations, we conducted a factorial analysis. Figure 1 shows that men with a good diet tend to be 55-76 years old, less educated, overweight, manual workers and living in a rural area. Women with a good diet were also 55-76 years old, less educated, wives of manual workers, but some women with a good diet belonged to the upper social class (Fig. 2). They had an ideal BMI and tend to live more frequently by the Mediterranean shore. Men with a poor diet tend to be 20-34 years old, smokers, unemployed or students, using vitamin and mineral supplements. The situation was comparable for women for the first two factors. In addition, women with a poor diet tended to be obese and to drink over 3 glasses of wine per day. The medium-good DQI correspondence for men is found with subjects who have an ideal BMI, drink either moderately or over 3 glasses of wine per day but are nonsmokers, and live in small towns or rural areas. For women with a medium-good DQI, the correspondence is restricted to moderate wine drinkers, and also found among inhabitants of larger towns $(>10000-<100000$ inhabitants). Both men and women with a medium-to-poor DQI are 35-54 years old, live in the urban area and tend to be better educated. The men tended to be non-drinkers and obese, whereas the women tend to be abnormally lean, with no correspondence with wine consumption.

Spearman correlations were computed between the biomarkers and the scores of DQI (carotene: -0.12 , $P=0.016$; vitamin E: $-0.20, P<0.05$; eicosapentaenoic acid (EPA): $-0.30, P<0.001$; docosahexaenoic acid (DHA) $-0.28, \quad P<0.001)$. The coefficient obtained

Table 1 Construction of the score

\begin{tabular}{lccccccc}
\hline Scores & SFA (\%energy) & Cholesterol $(\mathrm{mg})$ & Meats $(\mathrm{g})$ & Olive oil $(\mathrm{ml})$ & Fish $(\mathrm{g})$ & Cereals $(\mathrm{g})$ & Vegetables + fruit $(\mathrm{g})$ \\
\hline 0 & $<10$ & $<300$ & $<25$ & $>15$ & $>60$ & $>300$ \\
1 & $10-13$ & $300-400$ & $25-125$ & $15-5$ & $60-30$ & $300-100$ & $>700$ \\
2 & $>13$ & $>400$ & $>125$ & $<5$ & $<30$ & $<100$ & $700-400$ \\
\hline
\end{tabular}


Table 2 Intake median values of the subjects classified by the scores of the Diet Quality Index

\begin{tabular}{|c|c|c|c|c|c|c|c|c|c|}
\hline & & $\begin{array}{c}\text { SFA }{ }^{2} \\
\text { (\% energy) }\end{array}$ & $\begin{array}{l}\text { Cholesterol } \\
\text { (mg) }\end{array}$ & $\begin{array}{l}\text { Mammalian } \\
\text { foods (g) }\end{array}$ & Red meat & $\begin{array}{l}\text { Olive oil } \\
\text { (ml) }\end{array}$ & $\begin{array}{c}\text { Fish } \\
(\mathrm{g})\end{array}$ & Cereals (g) & $\begin{array}{c}\text { Fruit }+ \\
\text { vegetables }(\mathrm{g})\end{array}$ \\
\hline Good (1-4) & $N=89(9.2 \%)$ & 9 & 220 & 208 & 30 & 20 & 58 & 180 & 800 \\
\hline Medium-good (5-7) & $N=351(36.5 \%)$ & 12 & 230 & 240 & 50 & 10 & 31 & 178 & 645 \\
\hline Medium-poor $(8-10)$ & $N=428(44.4 \%)$ & 14 & 310 & 265 & 62 & 5 & 26 & 117 & 490 \\
\hline Poor $(10-14)$ & $N=96(9.9 \%)$ & 17 & 430 & 434 & 130 & 0.3 & 19 & 129 & 290 \\
\hline
\end{tabular}

between carotene and DQI-Ff was significant $(-0.17$, $P<0.05)$. It is interesting to note that the coefficients are higher for the correlation between the biomarkers and the DQI than for the correlation with the relevant intake (plasma vitamin $\mathrm{E}$ and vitamin $\mathrm{E}$ intake: 0.16, $P=0.06$; EPA and DHA and fish intake: $0.29, P<0.001$ and 0.23 , $P=0.005$, respectively), except for carotene (plasma carotene and carotene intake: $0.18, P=0.03$ ). The composite index made up of carotene, vitamin E, EPA and DHA showed the highest correlation coefficient: $-0.52(P=0.03)$ with the DQI, and $-0.58(P=0.01)$ with the DQI-f.

\section{Discussion}

Two aspects in our study bring a new insight to the way of capturing a multidimensional food behaviour. One is the construction of the DQI which differs somewhat from previously reported instruments ${ }^{6}$. All were developed in North European or North American populations. The recommendation of less than 30\% of energy from fat did not permit a good classification of our subjects. Thus, discriminating scores on the quality of fat appeared necessary and olive oil was introduced as a positive recommendation besides saturated fat as a negative recommendation. To complete the Mediterranean characteristics of the diet, we decided to integrate intake of red meat as a negative recommendation and intake of fish as a positive recommendation. The other new aspect highlighted by this study is the use of biomarkers to characterise a healthy or a poor diet. Plasma and/or urinary markers have been largely used to validate nutrient intake estimated by various questionnaires ${ }^{7}$ or specific food intakes ${ }^{8}$. However, such markers have not been used to characterise food patterns.

Our study shows a clear influence of age on dietary habits, for both men and women. In our study, older subjects had a better Med-DQI than younger subjects. One explanation may be that older people are more careful than young people about their health. Alternatively, it might be that older people continue to eat traditional Mediterranean food. This is supported by the association of good and medium-to-good diets with residence in rural

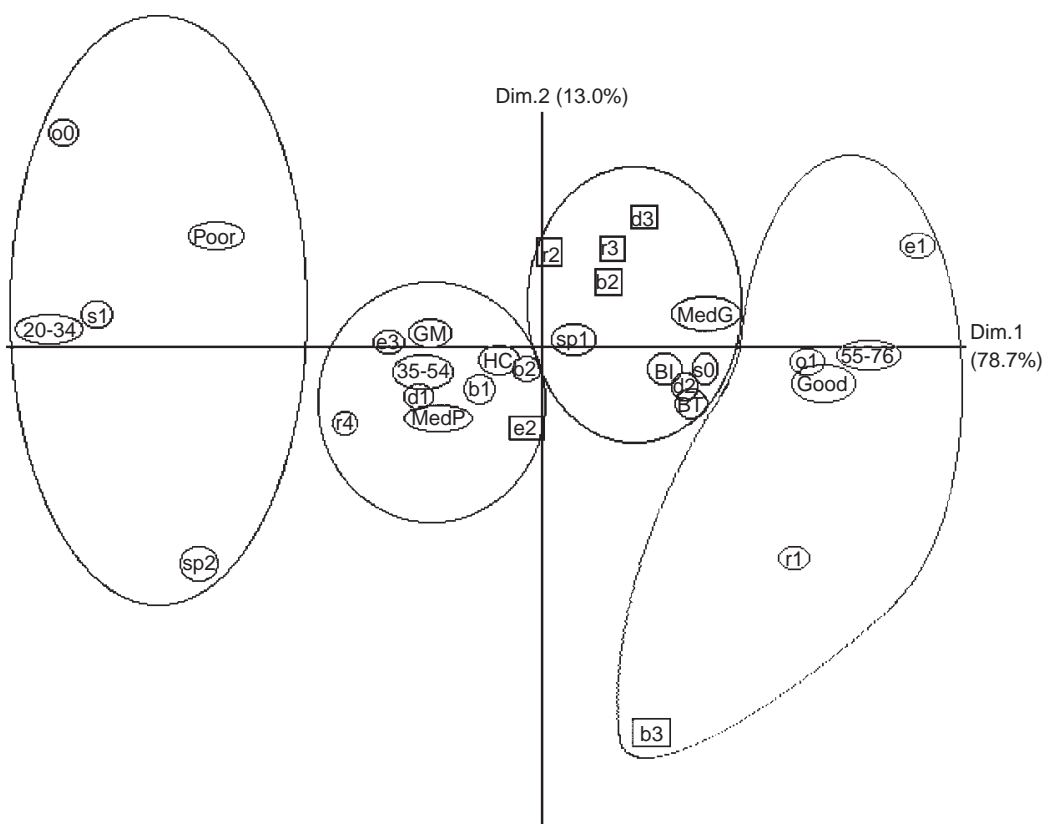

Fig. 1 Correspondence analysis in men. The variables contributing mainly to axis 1 are surrounded by a circle and those contributing mainly to axis 2 are surrounded by a square. MedP: medium-poor; MedG: medium-good. 20-34, 35-54, 55-76: age classes. b2: 18.5- < 25; b3: 25- < 30; b4: $\geq 30$. s0: non-smokers; s1: smokers. d1: light wine drinker; d2: moderate wine drinker; d3: heavy wine drinker. sp2: supplement use. e1: elementary school; e2: high school; e3, university. o0: without occupational activity (students, or unemployed); 01: workers in industry, farms or fisheries; r1: < 2000 inhabitants; r2 and BI: $2000-10000$ inhabitants; r4 and GM: >100 000 inhabitants. 


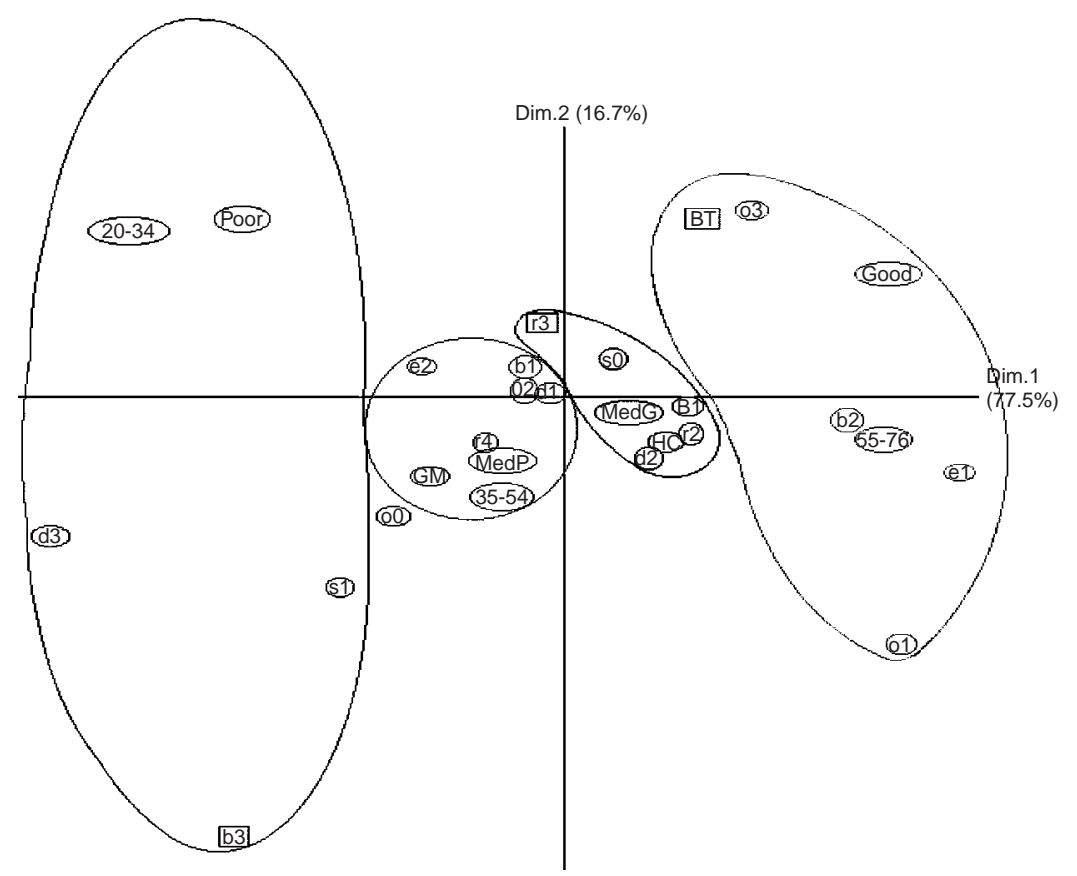

Fig. 2 Correspondence analysis in women. The variables contributing mainly to axis 1 are surrounded by a circle and those contributing mainly to axis 2 are surrounded by a square MedP: medium-poor; MedG: medium-good. 20-34, 35-54, 55-76: age classes. b1: $<18.5$; b2: 18.5- <25; b3: 25- < 30; b4: $\geq 30$. s1: smokers. d2: moderate wine drinker; d3: heavy wine drinker. e1: elementary school; e2: high school. 01: (spouse of) workers in industry, farms or fisheries; o3 executives or executives spouse. r2 and BI: $2000-10000$ inhabitants; r3: $>10000-<100000$ inhabitants. BT: sea-shore area; GM: >100 000 inhabitants.

areas or subregions. It has similarly been reported in another French study ${ }^{9}$ that traditional food habits are maintained among working class people.

With regard to wine drinking, men and women appeared to differ, since moderate and heavy drinking were associated with medium-good DQI and low drinking with medium-poor DQI in men, whereas in women heavy drinking was associated with poor DQI. Other findings were also different for men and women: factorial analysis showed different association with social classes. As noticed above, women from upper level social class, defined by their own or their spouse's professional activity, were associated with a good DQI, whereas men with a high level of education were associated with a medium-poor DQI. Overweight was associated with good DQI in men and poor DQI in women, obesity with medium-poor DQI in men and poor DQI in women. Factors such as obesity and drinking associated with poor diet in young women suggest a different behaviour than in young men, for which the associated factors, to be unemployed and to use supplements, may be more circumstantial. Our study confirms the association of smoking and poor dietary habits. In our region, drinking wine, even above 3 glasses per day, does not appear to be related to poor dietary habits, at least in men. In addition, young men with a good diet tend to drink more wine than the total sample, and when alcohol sources are considered, men with a poor diet show a significantly lower amount of wine intake than the total sample. This reflects the strong association between Mediterranean diet and wine drinking.

Correlation coefficients for carotene, vitamin $\mathrm{E}$ and $n-3$ fatty acids were as high or higher with the DQI than with the relevant nutrient or food. This has a first implication: if a decreased risk of disease is associated with a nutrient such as $\beta$-carotene, vitamin E or $n-3$ PUFA, it does not mean that this identified nutrient is responsible for the decreased risk, but it might be a whole food pattern of which the nutrient is a marker. The second one is that each one of these nutrients (except cholesterol) provided an estimation of the quality of the diet. However the correlation with DQI is higher when four biomarkers are used together, carotenes, vitamin $\mathrm{E}$ and $n-3$ fatty acids, as a composite index, especially when tobacco use is taken into the DQI. Smokers have lower plasma levels of $\beta$-carotene ${ }^{10}$ and lower concentrations of essential fatty acids in red blood cells ${ }^{11}$. Smokers have lower intake of polyunsaturated fat, vitamin $\mathrm{E}$ and $\beta$-carotene than nonsmokers $^{12}$. Beside a possible effect on food intake, this effect might be explained by the adverse effect of the tobacco on antioxidant status ${ }^{13}$.

In conclusion, it appears that: (1) fat intake cannot be considered as a whole when evaluating the diet quality since some fatty acids appear as risk factors of chronic degenerative diseases whereas others (or their food sources) seem to reduce the risk; (2) wine drinking is an integrated part of the Mediterranean diet; (3) subjects with 
$\alpha+\beta$-carotene levels $>1 \mathrm{mg} / \mathrm{l}$, vitamin $\mathrm{E}>30 \mathrm{mg} / \mathrm{l}$, EPA $>0.65 \%$ and DHA $>4 \%$ of fatty acids in erythrocytes are likely to have a good diet; (4) diet might only influence high levels of cholesterol $(>5 \mathrm{mM} / \mathrm{l})$, and that only very low levels (between 0.8 and $5 \mathrm{mM} / \mathrm{l}$ ) might be associated with a good diet in a non-smoking person; (v) finally our study shows the deleterious effect of tobacco use on blood nutrients which might increase the risk of some chronic degenerative diseases.

More generally, it can be said that Med-DQI is a good instrument to identify groups at risk with regard to nutritional quality. It can be improved by including separate scores for diversity, fruit and vegetables, raw and cooked vegetables, legumes and lignans as biomarkers of fibre intake.

\section{Acknowledgements}

The Languedoc-Roussillon Regional Council is gratefully acknowledged for financial support.

\section{References}

1 Gerber M, Scali J, Michaud A, Durand M, Astre C, Dallongeville J, et al. Profiles of a healthy diet and its relationship with biomarkers in a population sample from Mediterranean Southern France. Journal of the American Dietetic Association 2000; 100: 1164-71.

2 Trichopoulou A, Kouris-Blazos A, Wahlqvist M, Gnardellis C, Lagiou P, Polychronopoulos E, et al. Diet and overall survival in elderly people. British Medical Journal 1995; 311: 1457-60.

3 Siari S, Scali J, Richard A, Tretarre B, Daures JP, Padilla M, et al. Subregional variations of dietary consumption and incidences of cancer in Southern France. In: Riboli, Lambert, eds. Nutrition and Lifestyle: Opportunities for Cancer
Prevention. IARC Scientific Publications No. 156. Lyon: IARC, 2002; 127-9.

4 Gerber M. The comprehensive approach to diet: a critical review. Journal of Nutrition 2001; 131: S3051-5.

5 Bonifacj C, Gerber M, Scali J, Daurès JP. Comparison of dietary assessment methods in a Southern French population: use of weighed records, estimated-diet records and a food-frequency questionnaire. European Journal of Clinical Nutrition 1997; 51: 217-31.

6 Patterson RE, Haines PS, Popkin BM. Diet quality index: capturing a multidimensional behavior. Journal of the American Dietetic Association 1994; 94: 57-64.

7 Hunter D. Biochemical indicators of dietary intake. In: Willett W, ed. Nutritional Epidemiology. New York: Oxford University Press, 1990; 143-216.

8 Drewnowski A, Rock CL, Henderson SA, Shore AB, Fischler C, Galan P, et al. Serum beta-carotene and vitamin C as biomarkers of vegetable and fruit intakes in a communitybased sample of French adults. American Journal of Clinical Nutrition 1997; 65: 1796-802.

9 Poulain JP. Mutation des pratiques et nouvelles formes d'équilibre alimentaire. In: Agropolis, ed. Actes des Iv $v^{\text {emes }}$ rencontres du Réseau Agronomique Méditerranéen, Avignon, Agroparc, 1998; 77-93.

10 Saintot M, Astre C, Scali J, Gerber M. Within-subjects seasonal variation and determinants of inter-individual variations of plasma beta-carotene. International Journal for Vitamin and Nutrition Research 1994; 65: 169-74.

11 Brown KM, Morrice PC, Duthie GG. Erythrocyte membrane fatty acid composition of smokers and non-smokers: effects of vitamin E supplementation. European Journal of Clinical Nutrition 1998; 52: 145-50.

12 Dallongeville J, Marecaux N, Fruchart JC, Amouyel P. Cigarette smoking is associated with unhealthy patterns of nutrient intake: a meta-analysis. Journal of Nutrition 1998; 128: $1450-7$.

13 Marangon K, Herbeth B, Lecomte E, Paul-Dauphin A, Grolier $\mathrm{P}$, Chancerelle Y, et al. Diet, antioxidant status, and smoking habits in French men. American Journal of Clinical Nutrition 1998; 67: 231-9. 